\title{
Guía PARA EL DISEÑO DE UN PLAN ESTRATÉGICO DE MARKETING PARA EL INCREMENTO DE PROFESIONALES MUJERES EN LAS TECNOLOGÍAS DE LA INFORMACIÓN
}

\author{
Nadia Katherine Rodríguez Rodríguez \\ Nrodrigu@ulima.edu.pe \\ Universidad de Lima. Lima, Perú \\ Juan Manuel Gutiérrez Cárdenas \\ Jmgutier@ulima.edu.pe \\ Universidad de Lima. Lima, Perú
}

El presente trabajo busca mostrar la situación de una carrera de pregrado relacionada con las tecnologías de la información y el bajo número de mujeres entre su alumnado; en contraste con las grandes oportunidades laborales y de movilidad social que ofrece esta carrera, tanto en el ámbito nacional como internacional. Las empresas de hoy, en efecto, requieren profesionales de especialidades vinculadas a la informática, y, más aún, procuran la diversidad entre sus colaboradores. Este trabajo también pretende mostrar un marco conceptual relacionado con una metodología para elaborar un plan estratégico de marketing con el fin de incrementar la cantidad de mujeres en dichas carreras y, de esa forma, contribuir al balance de género en el Perú.

Palabras clave: profesionales mujeres / tecnologías de la información

\section{Abstract}

\section{Guide to design a strategic marketing plan to increase professional women in information technologies}

This work seeks to show the situation of an undergraduate career related to information technologies and the low population of women among its students; in contrast to the great job opportunities and social mobility offered by this career, both locally and internationally. Indeed, today's companies require professionals of careers linked to information technologies, and, moreover, seek diversity among their collaborators. This paper also aims to create a conceptual framework related to a marketing strategy and a methodology for the elaboration of a strategic marketing plan in order to increase the number of women in these careers. And also, to contribute to the gender balance in information technologies careers in Peru.

Keywords: Professionals women / information technologies 


\section{Introducción}

Si bien es cierto que la participación de las mujeres en la educación superior en el Perú ha mostrado un crecimiento importante a lo largo de los años (Díaz, 2008), el mismo crecimiento no se observa en las carreras de ingeniería (INEI, 2010). Específicamente, con respecto a las carreras universitarias de ingeniería relacionadas con las tecnologías de la información (en adelante TI), la población promedio de mujeres en comparación con la de hombres, tanto de universidades públicas como privadas, es de $25 \%$ y $75 \%$, respectivamente (INEI, 2010). El mismo fenómeno se observa también en otros países a escala mundial, donde la representación de mujeres en estas carreras fluctúa entre 10 \% y 40 \% (Galpin, 2002).

En la actualidad, los empleadores enfrentan escasez de talento con relación a los profesionales de las $\mathrm{TI}$ y, por lo tanto, han empezado a diversificar su capital humano buscando nuevas fuentes de talento (ManpowerGroup, 2017); adicionalmente, existe una tendencia a que se generen más puestos de trabajo en este campo, con posible demanda insatisfecha (Lacey y Wright, 2010). Sería beneficioso contar con más mujeres en el campo de las TI, debido a que, con sus marcadas características de género, como la imaginación y la capacidad inventiva, podrían aportar a su diversidad (Chatoney y Andreucci, 2009); por ejemplo, en el diseño de los productos y las innovaciones en el campo de las TI.

\section{Revisión de la literatura}

\subsection{Factores relacionados con el género y la elección de carreras vinculadas con las TI}

En algunas perspectivas teóricas, de acuerdo con los autores de las investigaciones revisadas de países distintos al Perú, se considera la relación entre el género femenino y su elección por carreras universitarias vinculadas a las TI y también a otras carreras universitarias afines con la ciencia y la ingeniería. Las teorías que tratan de explicar este fenómeno van desde la teoría del esencialismo de Aristóteles, basada puramente en las diferencias biológicas y psicológicas entre los géneros; la teoría del construccionismo social de Berger y Luckmann (1966), que propone que son los factores socioculturales y no necesariamente las fuerzas biológicas los que forman a los individuos y sus relaciones con la tecnología; la teoría de las diferencias individuales de género y TI de Trauth (2006), enfocada en las maneras en que los individuos, independientemente de su género, se distinguen en cuanto a su comportamiento, preferencias, talentos y elecciones en un contexto dado, y en que las relaciones entre los individuos y las tecnologías están basadas en tres constructos: identidad individual, influencia individual e influencia del contexto; hasta la teoría de la toma de decisiones de carrera de Hodkinson y Sparkes (1997), la cual afirma que en la toma de decisiones de carrera influyen las experiencias personales

y laborales del individuo; de manera parcial lo familiar y lo conocido; los antecedentes familiares, la cultura y las historias de vida. 
Las diferentes investigaciones coinciden en que la falta de interés de las mujeres por el campo de las TI no estaría relacionada con sus capacidades y habilidades, sino más bien con las influencias del contexto. La baja representación de las mujeres en el campo de las TI es un fenómeno global que debe analizarse y estudiarse en su propio contexto.

\subsection{Perspectiva de marketing}

Se debe promover la incorporación de más mujeres en las carreras vinculadas a las TI (Cohoon, 2002). Una de las maneras de promover esta incorporación es bajo una perspectiva de marketing estratégico, que permitiría definir y comprender con mayor precisión al segmento de consumidores objetivo, así como adaptar mejor sus servicios para que les sea atractivo a este mercado objetivo (Bock, Poole y Joseph, 2014).

\subsection{Estrategia de marketing}

Las estrategias de marketing que se puedan diseñar deben contemplar principalmente el desarrollo de un diagnóstico situacional, el cual proporciona información de entrada clave para el desarrollo de la estrategia de marketing. Sobre ello se tratará líneas más adelante, en la sección de metodología de elaboración de plan estratégico de marketing propuesta en este trabajo.

Según Kotler y Fox (1995), los stakeholders o interesados en las universidades pueden ser clasificados en dieciséis grupos: estudiantes matriculados, postulantes, docentes, padres, personal administrativo, egresados, proveedores, competidores, entidades gubernamentales, empresas, medios de comunicación, fundaciones, fideicomisarios, acreditadoras, comunidad local y público en general. Del mismo modo, Kotler y Fox (1995) toman el clásico modelo de marketing mix de las 4P: producto, precio, plaza y promoción, y lo adaptan para que funcione de manera más adecuada dentro de un contexto educacional, incorporándole tres elementos para generar el marketing mix de las 7P: proceso, infraestructura (physical evidence) y personas.

Rudd y Mills (2008) amplían la propuesta de las 7P de Kotler y proponen un marco conceptual con un modelo de mezcla de marketing de 9P para ser utilizado específicamente en la gestión de marketing estratégico de la educación superior, que incluye los siguientes elementos: producto, personas, precio, programación, promoción, posicionamiento, plaza, partnership (asociaciones) y packaging (empaquetamiento), como se ve la figura 1. Esta propuesta ayudaría a los encargados de esta tarea a desarrollar un plan estratégico de marketing con un enfoque específico a servicios. 
Figura 1. Modelo conceptual 9P

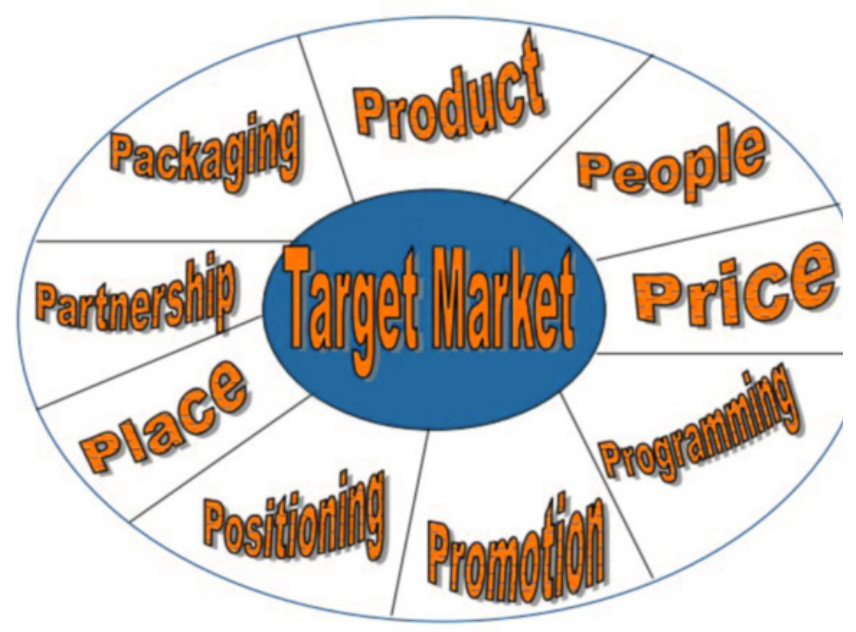

Fuente: Rudd y Mills (2008)

A continuación se describe cada elemento de la propuesta 9P de Rudd y Mills (2008).

\subsubsection{Producto}

Este primer ítem contempla todas las actividades, instalaciones, experiencias y servicios que provee la universidad, e incluye además los diversos programas académicos y sus planes de estudio; por ejemplo: servicio de cafetería, instalaciones deportivas, biblioteca, salones de clase, docentes, etcétera (Rudd y Mills, 2008). Además, considera aquellos "generadores de demanda"; por ejemplo: ubicación del campus, historia de la universidad, cultura e instalaciones recreacionales cercanas al campus universitario (Tourism Canada, Economic Planning Group y CommuniCorp, 1986).

La mejor universidad del mundo para el periodo 2017-2018 y en los últimos seis años, el Massachusetts Institute of Technology (MIT), de acuerdo a Quacquarelli Symonds Limited - QS (2017), tiene su campus ubicado en Cambridge, cuyo corazón muestra un grupo inicial de edificios interconectados para facilitar la interacción y comunicación entre las escuelas y departamentos del MIT. Además de los varios estilos arquitectónicos de los edificios, estos internamente están diseñados para apoyar los esfuerzos de investigación en curso del MIT en múltiples disciplinas. Estas instalaciones incluyen túneles aerodinámicos, aceleradores lineales, laboratorios de pruebas de robots $y$, actualmente en construcción, un centro de nanotecnología y de imágenes avanzadas de 18580 metros cuadrados. El campus se fusiona con varios barrios de Cambridge, donde la estrecha asociación de la industria y la experiencia de investigación han hecho de esta área la más innovadora del planeta (MIT, 2017). 


\subsubsection{Personas}

Se refiere a los empleados en la universidad y cómo estos pueden convertirse en herramientas de marketing sin incurrir en costos adicionales; por ejemplo cuando los docentes visitan colegios para ofrecer charlas vocacionales o cuando se difunden los logros de los docentes reconocidos como líderes en su especialidad (Rudd y Mills, 2008).

En el caso específico de las carreras relacionadas con las $\mathrm{TI}$, se necesita incrementar la población de docentes mujeres de TI que sean modelos inspiradores para las estudiantes (Cohoon y Lord, 2006), así como contar con docentes de ambos sexos comprometidos con el programa que apoyen de manera estable, sean automotivados y autogestionados, disfruten de enseñar a los estudiantes de pregrado, promuevan la comunicación e interacción estudiante-estudiante, estudiante-docente y con personas o grupos de soporte externos; muestren disposición para ser mentores de sus estudiantes; transmitan mensajes positivos y refuercen la confianza de las estudiantes mujeres, y que busquen oportunidades de involucrar a las estudiantes en grupos de investigación (Cohoon, 2002).

\subsubsection{Precio}

En el ámbito de la educación superior, el precio está asociado al monto por derechos de enseñanza que deben pagar los estudiantes para acceder al servicio educativo en la universidad; este constituye la principal fuente de ingresos de las instituciones (Filip, 2012). En el Perú, desde que el expresidente Alberto Fujimori promulgara la Ley de Promoción de la Inversión en la Educación (Decreto Legislativo 882, del 9 de noviembre de 1996), la cantidad de universidades privadas ha crecido de forma exponencial: "de las 142 universidades actuales, 91 instituciones son privadas (64 $\%)$, las cuales brindan educación al 75\% de la población estudiantil" (Fosca, 2016, p. 42). No obstante, este crecimiento carece del acompañamiento respectivo de control, aseguramiento y acreditación de la calidad; más aún en el caso de las universidades societarias o con fines de lucro, la rentabilidad ha tendido a primar a costa del sacrificio de la calidad de los recursos necesarios para brindar un servicio educativo idóneo (Fosca, 2016).

En consecuencia, existe mucha heterogeneidad en la forma en que las universidades peruanas privadas recaudan los derechos de enseñanza. Algunas casas de estudio mantienen valores escalonados con respecto a los derechos de enseñanza; estos se fijan, por ejemplo, de acuerdo con el colegio deprocedencia del estudiante o una evaluación económica de sus ingresos familiares. Adicionalmente, algunas ofrecen ayuda financiera a los estudiantes mediante becas o préstamos. En Estados Unidos, los autores Lozano, Guido, Torres y Talbot (2000) argumentan 
que las instituciones de educación superior deben proporcionar ayuda financiera adecuada y realista para permitir que un grupo minoritario, como las mujeres latinas, puedan alcanzar un grado de educación superior. A través de estas ayudas, las mujeres latinas pueden reducir o eliminar las horas que utilizan en trabajar, y dedicarlas a estudiar, así como reducir el estrés ocasionado por las preocupaciones financieras. Otro estudio declara que es $15 \%$ más probable que los estudiantes latinos asistan a la universidad si es que reciben algún tipo de ayuda financiera (St. John y Noell, 1989).

\subsubsection{Programación}

Comprende todos los eventos especiales y actividades para expandir el servicio principal que se ofrece, como programas de educación continua, programas vespertinos o de fines de semana para personas que trabajan, programas en línea, etcétera; en suma, todas aquellas actividades que atraen al estudiante no tradicional (Rudd y Mills, 2008).

Algunos autores argumentan que las mujeres sienten que el ambiente en las clases en la universidad de profesiones de TI, donde la composición es mayoritariamente masculina, se torna poco acogedor, en algunos casos hostil y podría inhibirlas para desenvolverse bien académicamente (Ahuja, 2002; Nix, Perez-Felkner y Thomas, 2015; Sáinz y Eccles, 2012). Otro estudio que evalúa las diferencias desde una perspectiva de género con respecto a clases en línea proporciona pruebas convincentes de que los cursos en línea son de gran valor para los estudiantes no tradicionales, especialmente las mujeres estudiantes adultas con niños o responsabilidades familiares (Sullivan, 2001). Tomando en cuenta los estudios anteriores, las universidades podrían pensar en diseñar una programación de clases especial presencial o en línea que atraiga más a las mujeres al campo de las TI.

\subsubsection{Promoción}

Consiste en el abanico de actividades que se llevan a cabo para estimular el interés en el servicio brindado, y que realzan el reconocimiento de la marca. Incluye comunicación, venta, publicidad, reclutamiento y gestión de las ventas (Rudd y Mills, 2008).

Considera la estrategia push, la cual se concentra en los intermediarios para que, a través de ellos, se logre "empujar" el producto a los clientes, por ejemplo: establecer contacto con docentes de secundaria para que mediante talleres se les ayude a mejorar sus habilidades con las TI en diversos campos, así como sensibilizarlos con respecto a la importancia de incorporar más mujeres en este campo; con orientadores 
educacionales para explicarles las oportunidades que ofrecen las TI, y que con esa información ellos puedan aconsejar a las estudiantes para que elijan una carrera de Tl; y coordinar visitas a los colegios de profesionales exitosos en TI para que compartan sus experiencias en la universidad y en el ámbito laboral (Cohoon, 2002). Por otro lado, considera estrategias pull, donde se trabaja directamente con el estudiante a través de, por ejemplo, publicidad en un solo sentido. Los medios de comunicación juegan un rol importante en la percepción de las mujeres con respecto a las carreras relacionadas a las $\mathrm{Tl}$, al mostrar muchas veces que son carreras solo para los hombres o que los profesionales de TI son "nerds" con pocas habilidades sociales (Thomas y Allen, 2006). Por ello, es necesario diseñar una estrategia de comunicación más feminizada que pueda dar a conocer las oportunidades que brindan a las mujeres las profesiones vinculadas con las Tl; que permita romper con los estereotipos existentes, incentivándolas a elegir una profesión que contribuya a disminuir el desbalance de género que aqueja a este campo.

\subsubsection{Posicionamiento}

El posicionamiento es el lugar que ocupa la marca en la mente de los consumidores respecto de los productos de la competencia. Para lograrlo, debe establecerse un mantra de la marca, que es "una articulación de las características más definitorias de la marca y está estrechamente relacionado con otros conceptos, como la esencia de la marca y la promesa central de la marca" (Kotler y Keller, 2012, p. 284). Cuando un estudiante sabe exactamente qué recibirá de su experiencia en la universidad, será más probable que no deserte y que se encuentre satisfecho (Rudd y Mills, 2008).

Aaker y Shansby (1982) identificaron seis enfoques de posicionamiento: por atributo, por uso, por usuario, por categoría de producto, por precio-calidad y posicionamiento competitivo. Basados en estos seis enfoques, Harrison (2009) describe y propone ejemplos académicos para cada uno de ellos: el posicionamiento por atributo es el más utilizado y está basado en asociar la marca a un atributo específico, característica del producto o beneficio para el usuario, por ejemplo, el Columbia College de Chicago posiciona el atributo de la innovación en las artes con su lema de posicionamiento "crear ... cambiar". El posicionamiento por uso responde al ¿para qué sirve?, se puede nombrar a la Universidad Cornell, que se caracteriza por su especialización en administración hotelera. En el posicionamiento por usuario se encuentran, por ejemplo, aquellas universidades que brindan programas especiales en horarios de fin de semana para personas que trabajan. La Universidad de Phoenix, por ejemplo, aunque provee aprendizaje remoto, se posiciona en una categoría de producto - que no le corresponde directamente - como universidad frente a la comunidad. En cuanto al posicionamiento por precio-calidad, la Universidad de Michigan, también Ilamada "la Harvard del Medio Oeste" por su buena reputación 
en las escuelas de medicina y derecho, es también una de las universidades estatales más costosas. Finalmente, en el posicionamiento competitivo las marcas se asocian a un punto de referencia, como a un competidor; por ejemplo, una universidad se podría asociar con otra universidad de mayor prestigio para buscar posicionamiento.

Si bien es cierto que un posicionamiento por usuario podría ayudar a incrementar la cantidad de mujeres en una carrera universitaria de $\mathrm{Tl}$, se debe considerar que en términos de posicionamiento no se puede complacer a todos los segmentos, ya que se puede generar una percepción confusa en la mente de los consumidores. El posicionamiento efectivo requiere una singularidad percibida (la única marca asociada con la base de posicionamiento particular), la fuerza (una asociación fuerte y clara con la base de posicionamiento) y la prevalencia (en la que la mayoría de los consumidores objetivo son conscientes del posicionamiento de la marca) (Harrison, 2009).

\subsubsection{Plaza}

Este elemento está referido a cuándo, dónde y a través de quién será ofrecido el servicio a la venta; por ejemplo, las universidades suelen tener campañas intensivas en ciertos momentos del año que suelen coincidir con los tiempos en que los escolares se deciden por sus carreras profesionales; de igual manera, definir los canales directos (presenciales o virtuales) que se utilizarán para llevar a cabo la promoción de la carrera y si el servicio será ofrecido solamente por el staff de marketing o también participará el staff académico (Rudd y Mills, 2008).

Cohoon (2002) sugiere que a través del contacto con los profesores y orientadores vocacionales de los colegios se puedan coordinar actividades de reclutamiento en los mismos colegios o en el campus de las universidades, durante los periodos adecuados para los colegios, enfocándose en ayudar a los estudiantes a definir sus carreras. Asimismo, recomienda establecer contacto con la comunidad local para que, mediante sus canales de información, se difundan las oportunidades laborales que el campo de las TI ofrece a sus profesionales.

En el Perú, el Estado lanzó en el 2005 el portal "Ponte en Carrera", cuyo objetivo era superar la asimetría de información que existe en el sistema educativo universitario. Por esta vía, los estudiantes y sus familias pueden conocer los costos de las universidades y el salario promedio que obtendrían dependiendo de la carrera y la universidad que elijan. De esa manera se busca ayudarlos a decidir por la carrera y universidad que ofrezca el mayor retorno sobre su inversión en la educación. Este portal podría aprovecharse para incluir también las necesidades y oportunidades de diversidad de género que se presentan en los diversos campos profesionales. 


\subsubsection{Partnership}

Consiste en dos o más organizaciones que ofrecen servicios complementarios y comparten sus recursos y actividades de marketing; por ejemplo, los convenios entre instituciones nacionales e internacionales que permiten transferir créditos educativos. Estas asociaciones hacen muy atractivas a las instituciones educativas, por el abanico de opciones de intercambio a las que el estudiante puede acceder (Rudd y Mills, 2008).

En el Perú, una clara muestra de partnership es el llamado Consorcio de Universidades creado en 1996 y conformado por cuatro de las universidades peruanas privadas más prestigiosas del país: Universidad de Lima, Universidad del Pacífico, Universidad Cayetano Heredia y Pontifica Universidad Católica del Perú.

A continuación se exponen la misión, los valores y los fines del Consorcio de Universidades:

La misión del Consorcio es expandir el saber mediante el desarrollo de proyectos interdisciplinarios e interinstitucionales, el mejoramiento de los recursos humanos altamente calificados al servicio del país y la ejecución de acciones específicas de proyección social.

Para cumplir esta misión, se inspira en valores como la verdad, el respeto por la dignidad de las personas, la libertad de pensamiento, la responsabilidad social y el compromiso por el desarrollo.

Asimismo, los fines que se ha propuesto la institución son:

- Contribuir al perfeccionamiento institucional de las universidades mediante la formación de recursos humanos altamente calificados y el desarrollo de una cultura de autoevaluación de las actividades administrativas, de docencia, de investigación y de proyección social.

- Realizar proyectos interinstitucionales de investigación y de desarrollo en el marco de programas de proyección social universitaria. Para ello, prioriza la capacitación de los agentes de desarrollo y promueve alternativas viables de solución de la pobreza.

- Beneficiar mediante la transferencia del conocimiento no solo a profesores y alumnos de las universidades del Consorcio, sino también a las organizaciones de la sociedad civil y del Estado que así lo requieran. (Consorcio de Universidades, 2017a)

A través del Consorcio los estudiantes tienen la opción de cursar asignaturas en las cuatro universidades, además del acceso a otros beneficios, como uso de 
las bibliotecas, actividades académicas y culturales, conferencias, entre otros. El Consorcio fomenta la formación de grupos de investigación interdisciplinaria entre los docentes de las cuatro universidades (Consorcio de Universidades, 2017a). En el 2015, el Consorcio inició un programa doctoral en Investigación de la Gestión Estratégica, "un programa diseñado para investigadores y profesionales altamente competitivos que asumirán el liderazgo en la conducción del desarrollo sostenible e inclusivo de los próximos años" (PUCP, 2016) el que actualmente se encuentra en su segunda edición y bajo la administración de la Pontificia Universidad Católica del Perú.

\subsubsection{Packaging}

Consiste en empaquetar ciertos servicios por un solo precio; por ejemplo, a través de partnerships ofrecer prácticas preprofesionales como parte del programa educativo; esto le brindaría al estudiante una valiosa experiencia, además de la posibilidad de enriquecer su hoja de vida (Rudd y Mills, 2008).

En el Perú, el Consorcio de Universidades ofrece su programa de intercambio estudiantil, donde los alumnos pueden acceder a cursos de especialidad en cualquiera de las cuatro universidades al mismo costo de la universidad de origen, además del uso de la infraestructura, los servicios académicos y otros servicios universitarios de las cuatro casas de estudio, lo que permite una "experiencia educativa y formación integral que trasciende a los cursos de cada carrera o universidad específica" (Consorcio de Universidades, 2017b).

\section{Metodología propuesta para la elaboración de un Plan Estratégico de Marketing}

Se propone utilizar la metodología de Kotler y Keller (2012) mostrada en la figura 2, que consta de cuatro fases: diagnóstico situacional, estrategia de marketing, finanzas y controles. En la fase de estrategia de marketing se propone utilizar el marco conceptual de las 9P de Rudd y Mills (2008). A continuación, se detalla cada fase:

\subsection{Fase 1: Diagnóstico situacional}

Se mostrará un análisis resumen del mercado, describiendo de manera específica la demografía del mercado de jóvenes, quienes son los que mayormente postulan a las carreras universitarias; los competidores, es decir aquellas otras carreras universitarias que compiten con carreras relacionadas a las $\mathrm{TI}$, así como las necesidades y tendencias del mercado. 
Figura 2. Fases en la elaboración de un plan estratégico de marketing

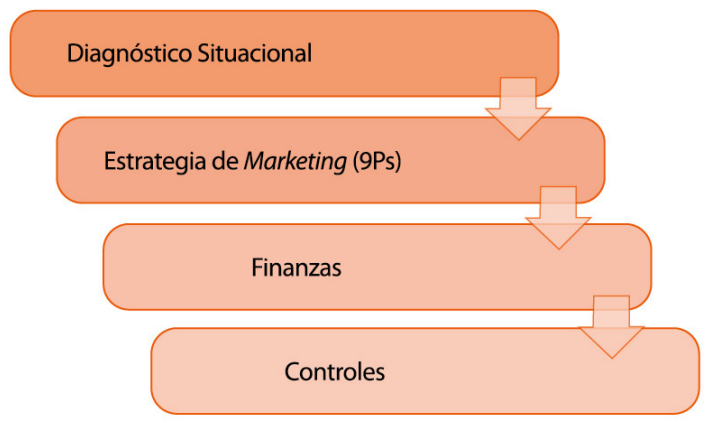

Fuente: Adaptado de Kotler y Keller (2012)

Para el diagnóstico se realizará un análisis del mercado.

\subsubsection{Demografía del mercado}

La cantidad de jóvenes de nuestro país que se encuentran en un rango de edad de 15 a 29 años asciende a 8377000 , que representa el $27 \%$ del total de la población peruana, de los cuales 4136000 son mujeres y 4241000 son hombres (INEI, 2015); es decir, existe casi un equilibrio en este segmento de población en cuanto a género se refiere.

La población económicamente activa en el rango de edad mencionado significa el $60,5 \%$, del cual $92,4 \%$ se encuentra trabajando. El 19,4\% de estos jóvenes que trabajan han obtenido educación superior universitaria (INEI, 2015), es decir, un poco más de novecientos mil jóvenes.

En el 2010, el panorama de ingresantes y postulantes a universidades públicas y privadas por género, de acuerdo a la ANR (2011), se muestra así: postularon a universidades públicas 309315 jóvenes, de los cuales 145304 (47\%) fueron mujeres, y 163911 (53\%), hombres; postularon a universidades privadas 239144 jóvenes, de los cuales 120857 (51\%) fueron mujeres y 118287 (49\%), hombres. Los ingresantes a universidades públicas fueron 63900 jóvenes, de los cuales 28798 (45,1\%) fueron mujeres y 35102 (54,9\%), hombres; ingresaron a universidades privadas 194541 jóvenes, de los cuales 98942 (50,9\%) fueron mujeres y 95599 (49,1\%), hombres. De acuerdo a estas cifras, se puede inferir, por un lado, que en el 2010 hubo un total de 138421 mujeres que postularon a universidades públicas y privadas, pero no obtuvieron una vacante de ingreso; por otro lado, existe casi un equilibrio en hombres y mujeres con respecto a los ingresantes a las universidades. Sin embargo, como se ha mencionado anteriormente, se observa un desequilibrio específicamente en las carreras relacionadas con las tecnologías de la información. 


\subsubsection{Competidores}

De acuerdo con cifras del último censo universitario nacional realizado por el INEI (2010), existe un total de 1677 carreras universitarias en todo el país, de las cuales 107 están relacionadas con las tecnologías de la información; es decir, solo un $6,4 \%$ del total. De las 107 carreras universitarias relacionadas con las $\mathrm{TI}, 46$ se encuentran en Lima metropolitana, es decir, casi un $43 \%$ de todas las carreras universitarias relacionadas con las TI ofrecidas en el Perú están concentradas en la capital. Es importante mencionar que de las carreras universitarias relacionadas con las TI, 8 pertenecen a universidades públicas. Si bien es cierto que el resto de carreras pertenece a las universidades privadas, un número reducido de estas son licenciadas, acreditadas y consideradas de prestigio.

\subsubsection{Necesidades del mercado}

Todos los jóvenes peruanos, hombres y mujeres, necesitan una educación superior de calidad que sea brindada por instituciones licenciadas y acreditadas, carreras que sean pertinentes con las necesidades del país, permitan una alta empleabilidad, ofrezcan transferencia de conocimientos y oportunidades en otras partes del mundo, y que brinden excelentes condiciones de trabajo más allá de las económicas.

Algunos estudios muestran que las carreras universitarias relacionadas con las $\mathrm{TI}$ son atractivas para los postulantes en general. Las carreras de ingeniería de sistemas e ingeniería informática ocupan los puestos 6 y 13, respectivamente, dentro del ranking de las 20 carreras más demandadas por los postulantes (Piscoya, 2008); sin embargo, como se ha manifestado, dichas carreras no parecen ser tan atractivas para el segmento de mujeres.

Por otra parte, la demanda laboral de egresados de carreras universitarias relacionadas con las $\mathrm{TI}$ en el Perú, tales como ingeniería de sistemas e ingeniería informática, ocupan los puestos 4 y 6 , respectivamente, en el ranking de las 20 profesiones más demandadas por las grandes empresas (Piscoya, 2008); sin embargo, los empleadores no logran satisfacer su demanda de profesionales de TI. Adicionalmente, de acuerdo con la última encuesta de escasez de talento de ManpowerGroup (2017), el $46 \%$ de empresas tienen dificultades para cubrir sus vacantes, siendo los puestos relacionados con las TI los más difíciles de cubrir. En el ámbito internacional, fuentes estadounidenses afirman que solamente el $25 \%$ de los puestos de trabajo relacionados con las TI son ocupados por mujeres (Nacional Center for Women \& Information Technology - NCWIT, 2017). 


\subsubsection{Tendencias del mercado}

Desde hace algunos años, varias instituciones internacionales han tomado interés en la escasez de talento femenino en los campos de las TI. El sitio web de Computer Science Online consolida un amplio directorio de instituciones estadounidenses que vienen desarrollando campañas estratégicas para promover e incrementar la participación de las mujeres en el campo de las TI (http://www. computerscienceonline.org).

El estudio sobre ocupaciones al 2018 en Estados Unidos proyecta un incremento de plazas laborales de aproximadamente $22 \%$ en profesiones relacionadas con las TI (Lacey y Wright, 2010). Ante la escasez de talento, algunas empresas peruanas han empezado a desarrollar estrategias para buscar nuevas fuentes de talento, por ejemplo, el segmento de mujeres (ManpowerGroup, 2015).

Con la información proveniente del análisis del mercado se debe elaborar o revisar la misión de la organización y desarrollar un análisis FODA, el cual permitirá identificar las fortalezas y debilidades claves de la carrera relacionada con las $\mathrm{TI}$ objeto de estudio, así como las oportunidades y amenazas a las que se enfrenta; lo cual, en conjunto, brindará la información clave para la formulación de los objetivos estratégicos de marketing con sus respectivos indicadores (Kotler y Keller, 2012).

\subsection{Fase 2: Estrategia de marketing}

La segunda fase consiste en el desarrollo de la estrategia de marketing. Antes de todo, se deberán trazar sus objetivos:

\section{Tabla 1. Ejemplo de objetivos e indicadores}

\begin{tabular}{c|l|l}
\hline $\mathbf{N} .^{\circ}$ & \multicolumn{1}{|c}{ Objetivo } & \multicolumn{1}{|c}{ Indicador } \\
\hline 1 & Incrementar la cantidad de alumnas en la carrera & $\begin{array}{l}\text { Nuevas alumnas } \\
\text { Total de alumnas }\end{array}$ \\
\hline 2 & Ser una carrera reconocida en el medio & Nivel de recordación \\
\hline
\end{tabular}

Luego, se deberá definir el mercado meta: si se elige un segmento demográfico basado en género, específicamente mujeres que están en los últimos años de secundaria o que ya egresaron de secundaria y están decidiendo su carrera profesional; es decir, están en un rango de 14 a 20 años, aproximadamente. Este rango de edad de 14 a 20 años pertenece a la generación $Z$, quienes han adoptado la tecnología casi desde su nacimiento, por lo que han generado dependencia hacia ella y se adaptan fácilmente a las nuevas tecnologías, se orientan mucho al mundo virtual y a los medios digitales, lo 
cual sería un factor que podría favorecer a la inclinación por una carrera relacionada con las tecnologías de la información.

Utilizando el marco conceptual de las 9P de Rudd y Mills (2008), desarrollado en la primera parte del presente artículo, se deberán definir y especificar adecuadamente cada uno de los elementos de las 9P: producto, personas, precio, programación, promoción, posicionamiento, plaza, partnership (asociaciones) y packaging (empaquetamiento).

\subsection{Fase 3: Finanzas}

Se revisarán los temas financieros para desarrollar un análisis de punto de equilibrio, pronósticos de ventas y de gastos, presupuestos, y se indicará cómo se vinculan estas actividades con la estrategia de marketing (Kotler y Keller, 2012).

\subsection{Fase 4: Controles}

Se trabajarán los controles donde, además de establecer métricas e indicadores, se evaluarán los efectos de las actividades y programas de marketing para poder realizar los cambios y ajustes necesarios, así como planes de contingencia para afrontar dificultades y riesgos (Kotler y Keller, 2012). Se proponen las siguientes métricas de control: crecimiento de postulantes mujeres, crecimiento de ingresantes mujeres, disminución de deserción de mujeres, conciencia de la marca a nivel de carrera, preferencia de la marca a nivel de carrera.

\section{Conclusiones y recomendaciones}

\subsection{Conclusiones}

i. Las estadísticas muestran un desequilibrio en la representación de mujeres en las carreras relacionadas con las tecnologías de la información en el Perú. Asimismo, los estudios muestran las grandes oportunidades que el campo laboral de las TI ofrece y a su vez la escasez de talento existente, lo que estaría llevando a las empresas a buscar nuevas fuentes de talento que permitan añadir diversidad a su talento humano.

ii. Se presenta el marco conceptual diseñado para organizaciones que ofrecen el servicio de educación superior desarrollado por Rudd y Mills (2008), como una evolución de la propuesta de las 7P de Kotler y que está basado en los elementos producto, personas, precio, programación, promoción, posicionamiento, plaza, partnership y packaging. Este modelo permitirá la formulación y utilización de estrategias y herramientas de marketing que podrían contribuir a incrementar la participación femenina en el campo educativo de las TI. 
iii. Se propone una metodología para la elaboración de un plan estratégico de marketing para promover la incorporación de más mujeres en este tipo de carreras, así como para el diseño de las estrategias correspondientes.

\subsection{Recomendaciones}

i. Se propone la utilización del modelo de Kotler para la elaboración de un plan estratégico de marketing, que contiene las siguientes fases: diagnóstico situacional, estrategia de marketing, finanzas y controles. En el caso de la fase específica relacionada con el desarrollo de la estrategia de marketing se propone utilizar el marco conceptual de las 9P de Rudd y Mills (2008), que presenta una mezcla de marketing para ser utilizada específicamente en la gestión de marketing estratégica de la educación superior.

ii. Se recomienda la elaboración del plan estratégico de acuerdo a la metodología propuesta, y su posterior implementación para realizar un seguimiento por lo menos en tres procesos de admisión, y analizar los resultados obtenidos. De esa manera se podrá comprobar si la metodología propuesta, y su adecuada implementación, contribuyen a superar el desbalance de género en la carrera relacionada con las $\mathrm{TI}$ de las universidades del Perú mediante la incorporación de un mayor número de mujeres a la especialidad mencionada.

\section{Referencias}

Aaker, D., y Shansby, J. (1982). Positioning your product. Business Horizons, 25(3), 56-62. DOI: 10.1016/0007-6813(82)90130-6

Ahuja, M. (2002). Women in the information technology profession: a literature review, synthesis and research agenda. European Journal of Information Systems, 11(1), 20-34. DOI: 10.1057/ palgrave/ejis/3000417

ANR. (2011). Datos estadísticos universitarios. Universidades 2011, Población universitaria 2010. Recuperado de http://censos.inei.gob.pe/cenaun/redatam_inei/doc/ESTADISTICA_ UNIVERSITARIAS.pdf

Berger, P. y Luckmann, T. (1966). The Social Construction of Reality: A Treatise in the Sociology of Knowledge. Nueva York, Estados Unidos: Doubleday/Anchor. DOI: 10.2307/323448

Bock, D., Poole, S., y Joseph, M. (2014). Does branding impact student recruitment: a critical evaluation. Journal of Marketing for Higher Education, 24(1), 11-21. DOI: 10.1080/08841241.2014.908454 
Chatoney, M., y Andreucci, C. (2009). How study aids influence learning and motivation for girls in technology education. International Journal of Technology and Design Education, 19(4), 393-402. DOI: 10.1007/s10798-009-9094-8

Cohoon, J. (2002). Recruiting and retaining women in undergraduate computing majors. ACM SIGCSE Bulletin - Women and Computing, 34(2), 48-52. DOI: 10.1145/543812.543829

Cohoon, J., y Lord, H. (2006). A Faculty Role in Women's Participating in Computing. En Encyclopedia of Gender and Information Technology (297-303). IGI Global. DOI: 10.4018/9781-59140-815-4.ch046

Consorcio de Universidades. (2017a). Nosotros. Recuperado de http://www.consorcio.edu.pe/ nosotros/

Consorcio de Universidades. (2017b). Programa de Intercambio Académico Estudiantil. Recuperado de http://www.consorcio.edu.pe/actividades/intercambioacademico/

Decreto Legislativo 882. (9 de noviembre de 1996). Ley de promoción de la inversión en la educación. Diario oficial El Peruano.

Díaz, J. (2008). Educación superior en el Perú: tendencias de la demanda y la oferta. En M., Benavides (Ed.), Análisis de programas, procesos y resultados educativos en el Perú: contribuciones empíricas para el debate (83-129). Lima, Perú: Grupo de Análisis para el Desarrollo. Recuperado de http://biblioteca.clacso.edu.ar/Peru/grade/20100405042637/analisis-2.pdf

Filip, A. (2012). Marketing theory applicability in higher education. Procedia-Social and Behavioral Sciences, 46, 912-916. DOI: 10.1016/j.sbspro.2012.05.223

Fosca, C. (2016). Sobre la gestión de las universidades y su financiamiento. En S. Pedraglio (Coord.), Dosier. Aproximaciones a la educación universitaria. Perú 2016 (36-51). Lima, Perú: Pontificia Universidad Católica del Perú.

Galpin, V. (2002). Women in Computing Around the World. ACM SIGCSE Bulletin, 34(2), 94-100. DOI: $10.1145 / 543812.543839$

Harrison, L. (2009). Strategic Positioning in Higher Education. Academy of Educational Leadership Journal, 13(1), 103-111.

Hodkinson, P., y Sparkes, A. (1997). Careership: a sociological theory of career decision making. British Journal of Sociology of Education, 18(1), 29-44. DOI: 10.1080/0142569970180102

Instituto Nacional de Estadística e Informática - INEI (2010). I/Censo Naciona/Universitario. [Sistema de consulta de datos]. Recuperado de http://censos.inei.gob.pe/cenaun/redatam_inei/ 
Instituto Nacional de Estadística e Informática - INEl. (11 de agosto del 2015). El $27 \%$ de la población peruana son jóvenes [Nota de prensa]. Recuperado de https://www.inei.gob.pe/ prensa/noticias/el-27-de-la-poblacion-peruana-son-jovenes-8547/

Kotler, P., y Fox, K. (1995). Strategic marketing for educational institutions (2. ${ }^{\text {a }}$ ed.). Nueva Jersey, Estados Unidos: Prentice-Hall.

Kotler, P., y Keller, K. (2012). Dirección de marketing (14. a ed.). México, D. F.: Pearson Educación.

Lacey, T., y Wright, B. (2010). Occupational employment projections to 2018. Monthly Labor Review, 82-123. Recuperado de https://www.bls.gov/opub/mlr/2009/11/art5full.pdf

Lozano, A., Guido, F., Torres, V., y Talbot, D. (2000). Latina College Students: Issues and Challenges for the 21st Century. Journal of Student Affairs Research and Practice, 37(3), 511-527. DOI: 10.2202/1949-6605.1111

ManpowerGroup. (2015). Encuesta de escasez de talento 2015. Recuperado de https://www. manpower.com.pe/mpintranet/publicaciones/3166-9795432090759.pdf

ManpowerGroup. (2017). Encuesta de escasez de talento. Resultados Perú 2016/2017. Recuperado de https://www.manpower.com.pe/mpintranet/publicaciones/4466-9358284738328.pdf

Massachusetts Institute of Technology - MIT. (2017). Mit Facts. The campus. Recuperado de http:// web.mit.edu/facts/campus.html

Nacional Center for Women \& Infrmation Technology - NCWIT. (2017). Women and Information Technology. By the Numbers. Recuperado de https://www.ncwit.org/sites/default/files/ resources/btn_03232016_web.pdf

Nix, S., Perez-Felkner, L., y Thomas, K. (2015). Perceived mathematical ability under challenge: a longitudinal perspective on sex segregation among STEM degree fields. Frontiers in Psychology, 6, 1-19. DOI: 10.3389/fpsyg.2015.00530

Piscoya, L. (2008). Formación universitaria vs. Mercado laboral II. Lima, Perú: Asamblea Nacional de Rectores del Perú / lesalc-Unesco.

Pontificia Universidad Católica del Perú - PUCP. (2016). Doctorado en Gestión Estratégica. Recuperado de http://posgrado.pucp.edu.pe/doctorado/gestion-estrategica/

Quacquarelli Symonds Limited - QS. (2017). QSWorld University Rankings. Recuperado de https:// www.topuniversities.com/university-rankings/world-university-rankings/2018 
Rudd, D., y Mills, R. (2008). Expanding Marketing principles for the sale of higher education. Contemporary Issues in Education Research, 1(3), 41-52. Recuperado de http://files.eric. ed.gov/fulltext/EJ1056381.pdf

Sáinz, M., y Eccles, J. (2012). Self-concept of computer and math ability: Gender implications across time and within ICT studies. Journal of Vocational Behavior, 80(2), 486-499. DOI: 10.1016/j.jvb.2011.08.005

St. John, E., y Noell, J. (1989). The effects of student financial aid on access to higher education: An analysis of progress with special consideration of minority enrollment. Research in Higher Education, 30(6), 563-581. DOI: 10.1007/BF00992391

Sullivan, P. (2001). Gender differences and the online classroom: male and female college students evaluate their experiences. Community College Journal of Research and Practice, 25(10), 805-818. DOI: 10.1080/106689201753235930

Thomas, T., y Allen, A. (2006). Gender Differences in Students' Perceptions of Information Technology as a Career. Journal of Information Technology Education, 5, 165-178. Recuperado de http://jite.org/documents/Vol4/JiteContentsVol5.pdf

Tourism Canada, Economic Planning Group, CommuniCorp, Inc (1986). Tourism is your business. marketing management: a program for Canada's tourism industry. Toronto, Canadá: Canadian Hotel \& Restaurant / Maclean Hunter / Tourism Canada and the Canadian Government Pub. Centre.

Trauth, E. (2006). Theorizing Gender and Information Technology Research. En Encyclopedia of Gender and Information Technology (1154-1159). IGI Global. DOI: 10.4018/978-1-59140815-4.ch182 